\title{
Front Matter: Volume 8427
}

, "Front Matter: Volume 8427," Proc. SPIE 8427, Biophotonics: Photonic Solutions for Better Health Care III, 842701 (23 May 2012); doi:

10.1117/12.940785

SPIE. Event: SPIE Photonics Europe, 2012, Brussels, Belgium 


\section{PROGRESS IN BIOMEDICAL OPTICS AND IMAGING}

Vol. 13, No. 40

\section{Biophotonics: Photonic Solutions for Better Health Care III}

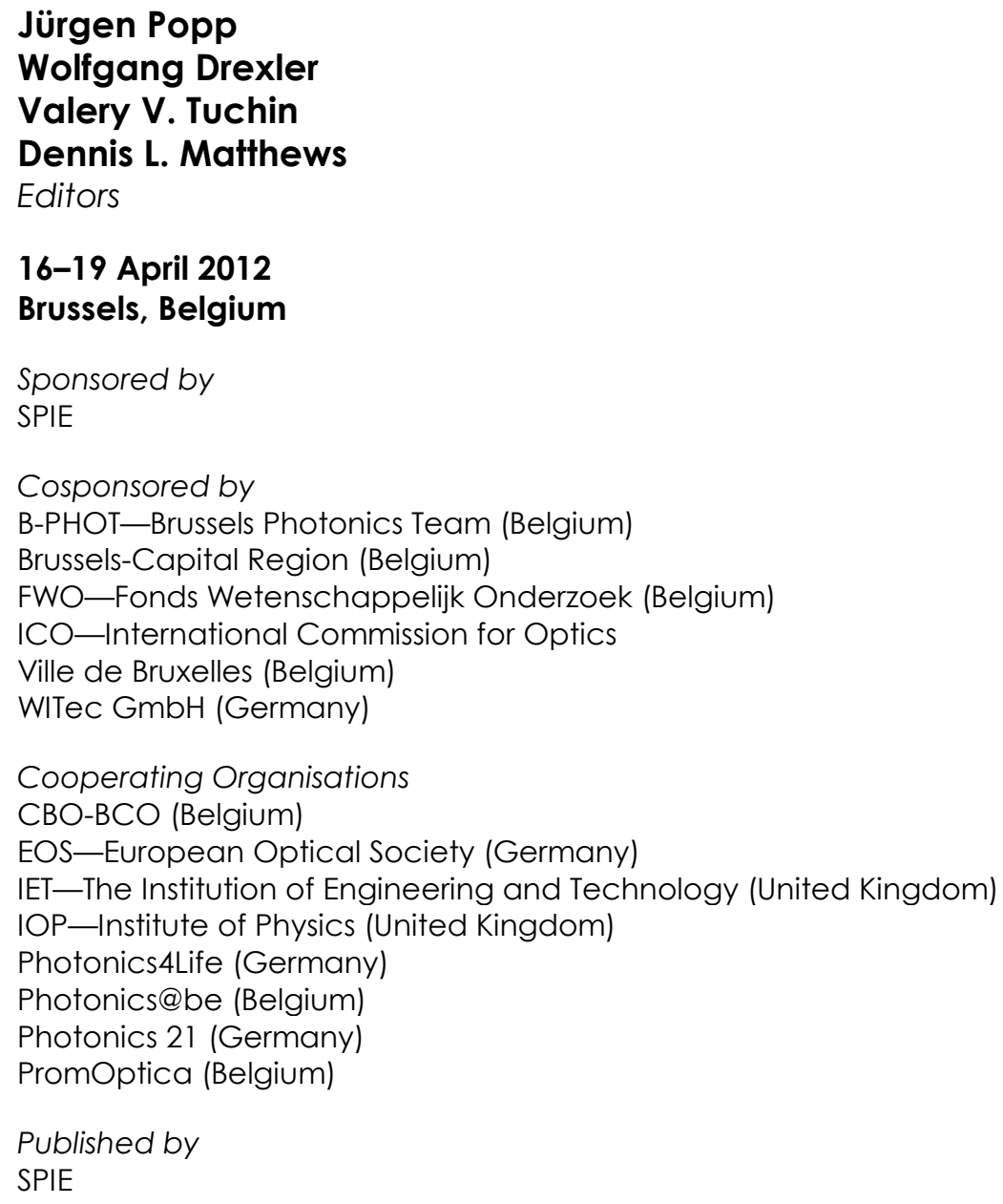


The papers included in this volume were part of the technical conference cited on the cover and title page. Papers were selected and subject to review by the editors and conference program committee. Some conference presentations may not be available for publication. The papers published in these proceedings reflect the work and thoughts of the authors and are published herein as submitted. The publisher is not responsible for the validity of the information or for any outcomes resulting from reliance thereon.

Please use the following format to cite material from this book:

Author(s), "Title of Paper," in Biophotonics: Photonic Solutions for Better Health Care III, edited by Jürgen Popp, Wolfgang Drexler, Valery V. Tuchin, Dennis L. Matthews, Proceedings of SPIE Vol. 8427 (SPIE, Bellingham, WA, 2012) Article CID Number.

ISSN 1605-7422

ISBN 9780819491190

Published by

SPIE

P.O. Box 10, Bellingham, Washington 98227-0010 USA

Telephone +1 3606763290 (Pacific Time) · Fax +1 3606471445

SPIE.org

Copyright (c) 2012, Society of Photo-Optical Instrumentation Engineers.

Copying of material in this book for internal or personal use, or for the internal or personal use of specific clients, beyond the fair use provisions granted by the U.S. Copyright Law is authorized by SPIE subject to payment of copying fees. The Transactional Reporting Service base fee for this volume is $\$ 18.00$ per article (or portion thereof), which should be paid directly to the Copyright Clearance Center (CCC), 222 Rosewood Drive, Danvers, MA 01923. Payment may also be made electronically through CCC Online at copyright.com. Other copying for republication, resale, advertising or promotion, or any form of systematic or multiple reproduction of any material in this book is prohibited except with permission in writing from the publisher. The CCC fee code is 1605 $7422 / 12 / \$ 18.00$.

Printed in the United States of America.

Publication of record for individual papers is online in the SPIE Digital Library.

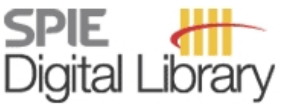

SPIEDigitallibrary.org

Paper Numbering: Proceedings of SPIE follow an e-First publication model, with papers published first online and then in print and on CD-ROM. Papers are published as they are submitted and meet publication criteria. A unique, consistent, permanent citation identifier (CID) number is assigned to each article at the time of the first publication. Utilization of CIDs allows articles to be fully citable as soon as they are published online, and connects the same identifier to all online, print, and electronic versions of the publication. SPIE uses a six-digit CID article numbering system in which:

- The first four digits correspond to the SPIE volume number.

- The last two digits indicate publication order within the volume using a Base 36 numbering system employing both numerals and letters. These two-number sets start with $00,01,02,03,04$, $05,06,07,08,09,0 A, 0 B \ldots$. 0Z, followed by 10-1Z, 20-2Z, etc.

The CID number appears on each page of the manuscript. The complete citation is used on the first page, and an abbreviated version on subsequent pages. Numbers in the index correspond to the last two digits of the six-digit CID number. 


\section{Contents}

xv Conference Committee

\section{MICROSCOPY I}

842703 Patterned illumination for analysing neuronal function in 3D [8427-02]

M. A. Go, C. Stricker, S. Redman, H. Bachor, V. R. Daria, The Australian National Univ. (Australia)

842704 Estimation of single cell volume from 3D confocal images using automatic data processing [8427-03]

A. Chorvatova, International Laser Ctr. (Slovakia); M. Cagalinec, International Laser Ctr. (Slovakia) and Comenius Univ. (Slovakia); A. Mateasik, D. Chorvat, Jr., International Laser Ctr. (Slovakia)

842706 New imaging technique using degree of polarization for the study of polarimetric properties for non-invasive biomedical diagnostic [8427-05]

I. C. Buscemi, S. Guyot, J. Lemoine, Lissy Lab., CNRS, Univ. Paris-Est Créteil (France)

\section{MICROSCOPY II}

842709 Self-interference digital holographic microscopy for live cell imaging [8427-08]

B. Kemper, S. Dartmann, F. Schlichthaber, A. Vollmer, S. Ketelhut, G. von Bally, Univ. Münster (Germany)

$8427 \mathrm{OA}$ Optical tweezers induced photodamage in living cells quantified with digital holographic phase microscopy [8427-09]

Á. Barroso Peña, B. Kemper, M. Woerdemann, A. Vollmer, S. Ketelhut, G. von Bally, C. Denz, Univ. Münster (Germany)

8427 OB Quantitative measurement of absolute cell volume and intracellular integral refractive index (RI) with dual-wavelength digital holographic microscopy (DHM) [8427-10] D. Boss, Ecole Polytechnique Fédérale de Lausanne (Switzerland); J. Kuehn, Ctr. de Neurosciences Psychiatriques, CHUV (Switzerland); C. Depeursinge, Ecole Polytechnique Fédérale de Lausanne (Switzerland); P. J. Magistretti, P. Marquet, Ecole Polytechnique Fédérale de Lausanne (Switzerland) and Ctr. de Neurosciences Psychiatriques, CHUV (Switzerland) 
8427 OC Membrane permeable luminescent metal complexes for cellular imaging (Invited Paper) [8427-11]

U. Neugebaver, Universitätsklinikum Jena (Germany), Institute of Photonic Technology (Germany), and Dublin City Univ. (Ireland); L. Cosgrave, Y. Pellegrin, Dublin City Univ. (Ireland); M. Devocelle, Royal College of Surgeons in Ireland (Ireland); R. J. Forster, T. E. Keyes, Dublin City Univ. (Ireland)

$8427 \mathrm{OE} \quad$ Microring resonator arrays for multiparameter biochemical analysis [8427-13] P. Lützow, D. Pergande, D. Gausa, S. Huscher, H. Heidrich, Fraunhofer-Institut für Nachrichtentechnik Heinrich-Hertz-Institut (Germany)

\section{OPTICAL SENSORS II}

8427 0l Layered polymer: inorganic composite waveguides for biosensor applications [8427-17] J. Hiltunen, VTT Technical Research Ctr. of Finland (Finland); M. Wang, Univ. of Oulu (Finland); C. Liedert, S. Aikio, N. Masuda, VTT Technical Research Ctr. of Finland (Finland); S. Pearce, M. Charlton, Univ. of Southampton (United Kingdom); P. Karioja, VTT Technical Research Ctr. of Finland (Finland)

8427 0J Development of a FBG probe for non-invasive carotid pulse waveform assessment [8427-18] C. Leitão, Univ. de Aveiro (Portugal); L. Bilro, N. Alberto, Instituto de Telecomunicações (Portugal); P. Antunes, Univ. de Aveiro (Portugal) and Instituto de Telecomunicações (Portugal); H. Lima, Univ. de Aveiro (Portugal); P. S. André, R. Nogueira, Instituto de Telecomunicações (Portugal); J. L. Pinto, Univ. de Aveiro (Portugal) and Instituto de Telecomunicações (Portugal)

8427 OK A minimally invasive chip based near infrared sensor for continuous glucose monitoring [8427-19]

L. Ben Mohammadi, S. Sigloch, I. Frese, V. Stein, K. Welzel, F. Schmitz, T. Klotzbücher, Institut für Mikrotechnik Mainz GmbH (Germany)

$8427 \mathrm{OL}$ Photoplethysmography system for blood pulsation detection in unloaded artery conditions [8427-20]

A. Grabovskis, Z. Marcinkevics, O. Rubenis, U. Rubins, V. Lusa, Univ. of Latvia (Latvia)

\section{OPTICAL MICROMANIPULATION I}

8427 OM Application of laser tweezers Raman spectroscopy techniques to the monitoring of single cell response to stimuli (Invited Paper) [8427-21]

J. W. Chan, R. Liu, D. L. Matthews, Univ. of California, Davis (United States)

8427 ON Video-based analysis of the rotational behaviour of rod-shaped, self-propelled bacteria in holographic optical tweezers [8427-22]

L. Dewenter, C. Alpmann, M. Woerdemann, C. Denz, Univ. Münster (Germany) 
8427 OR Towards gene therapy based on femtosecond optical transfection (Invited Paper) [8427-26] M. Antkowiak, M. L. Torres-Mapa, Univ. of St. Andrews (United Kingdom); J. McGinty, M. Chahine, L. Bugeon, A. Rose, A. Finn, Imperial College London (United Kingdom); S. Moleirinho, Univ. of St. Andrews (United Kingdom); K. Okuse, M. Dallman, P. French, S. E. Harding, Imperial College London (United Kingdom); P. Reynolds, F. Gunn-Moore, K. Dholakia, Univ. of St. Andrews (United Kingdom)

8427 OS Noncontact microsurgery and micromanipulation of living cells with combined system femtosecond laser scalpel-optical tweezers [8427-27]

I. V. Il'ina, D. S. Sitnikov, A. V. Ovchinnikov, M. B. Agranat, Joint Institute for High Temperatures (Russian Federation); Y. V. Khramova, M. L. Semenova, Lomonosov Moscow State Univ. (Russian Federation)

8427 OT Photonic crystal cavities for resonant evanescent field trapping of single bacteria [8427-28] T. van Leest, J. Heldens, Delft Univ. of Technology (Netherlands); B. van der Gaag, KWR Watercycle Research Institute (Netherlands); J. Caro, Delft Univ. of Technology (Netherlands)

8427 OU Towards a laser-integrated module for marker-free sorting of micrometer-sized particles in microfluidic channels [8427-29]

W. Schwarz, A. Bergmann, A. J. Márquez del Pino, D. Wahl, Ulm Univ. (Germany); D. Rimpf, T. Mappes, Karlsruhe Institute of Technology (Germany); R. Michalzik, Ulm Univ. (Germany)

8427 OV Optical trapping detects colored noise in the fluctuations of an extended DNA molecule [8427-30]

I. A. Martínez, S. Raj, ICFO - Institut de Ciencies Fotòniques (Spain); D. Petrov, ICFO - Institut de Ciències Fotòniques (Spain) and Institució Catalana de Recerca i Estudis Avançats (Spain)

\section{ADVANCED MICROSCOPIC AND SPECTROSCOPIC METHODS I}

8427 OY Two-photon-excited autofluorescence and second-harmonic generation microscopy for the visualization of penetration of $\mathrm{TiO}_{2}$ and $\mathrm{ZnO}$ nanoparticles into human tooth tissue ex vivo (Best Student Paper Award) [8427-33]

N. A. Trunina, N.G. Chernyshevsky Saratov State Univ. (Russian Federation); A. P. Popov, Univ. of Oulu (Finland); J. Lademann, Charité Universitätsmedizin Berlin (Germany); V. V. Tuchin, N.G. Chernyshevsky Saratov State Univ. (Russian Federation) and Univ. of Oulu (Finland); R. Myllylä, Univ. of Oulu (Finland); M. E. Darvin, Charité Universitätsmedizin Berlin (Germany)

$84270 Z$ Non-invasive label-free investigation and typing of head and neck cancers by multimodal nonlinear microscopy [8427-34]

T. Meyer, N. Vogler, Institut für Photonische Technologien e.V. (Germany); B. Dietzek, Institut für Photonische Technologien e.V. (Germany) and Friedrich-Schiller-Univ. Jena (Germany); D. Akimov, Institut für Photonische Technologien e.V. (Germany); J. Inhestern, O. Guntinas-Lichius, Universitätsklinikum Jena (Germany); J. Popp, Institut für Photonische Technologien e.V. (Germany) 
842710 Discrimination of skin diseases using the multimodal imaging approach [8427-35]

N. Vogler, S. Heuke, D. Akimov, I. Latka, Institut für Photonische Technologien e.V.

(Germany); F. Kluschke, H.-J. Röwert-Huber, J. Lademann, Charité Universitätsmedizin Berlin

(Germany); B. Dietzek, J. Popp, Institut für Photonische Technologien e.V. (Germany) and

Friedrich-Schiller-Univ. Jena (Germany)

\section{ADVANCED MICROSCOPIC AND SPECTROSCOPIC METHODS II}

842712 Force and Raman spectroscopy of single red blood cell [8427-37]

S. Raj, M. Wojdyla, M. Marro Sanchez, ICFO - Institut de Ciències Fotòniques (Spain);

D. Petrov, ICFO - Institut de Ciències Fotòniques (Spain) and Institució Catalana de Recerca i Estudis Avançats (Spain)

842713 Use of Raman microspectroscopy to score the malignancy of breast cancer cells [8427-38] C. Nieva, IDIBELL - Institut d'Investigació Biomèdica de Bellvitge (Spain) and ICFO - Institut de Ciències Fotòniques (Spain); M. Marro, ICFO - Institut de Ciències Fotòniques (Spain); R. Sanz-Pamplona, IDIBELL - Institut d'Investigació Biomèdica de Bellvitge (Spain); N. Santana-Codina, IDIBELL - Institut d'Investigació Biomèdica de Bellvitge (Spain) and Univ. Autònoma de Barcelona (Spain); S. Rao, ICFO - Institut de Ciències Fotòniques (Spain); D. Petrov, ICFO - Institut de Ciències Fotòniques (Spain) and ICREA - Institució Catalana de Recerca i Estudis Avançats (Spain); A. Sierra, IDIBELL - Institut d'Investigació Biomèdica de Bellvitge (Spain)

842714 Double optical fibre-probe device for the diagnosis of melanocytic lesions [8427-39] R. Cicchi, A. Cosci, S. Rossari, V. De Giorgi, D. Kapsokalyvas, D. Massi, F. S. Pavone, Univ. degli Studi di Firenze (Italy)

842715 Detection of neuroinflammation through the retina by means of Raman spectroscopy and multivariate analysis [8427-40]

M. Marro, ICFO - Institut de Ciències Fotòniques (Spain); A. Taubes, P. Villoslada, IDIBAPS Institut d'Investigacions Biomèdiques Agust Pi i Sunyer (Spain); D. Petrov, ICFO - Institut de Ciències Fotòniques (Spain) and ICREA - Institució Catalana de Recerca i Estudis Avançats (Spain)

\section{ADVANCED MICROSCOPIC AND SPECTROSCOPIC METHODS III}

842719 Load bearing studies of single DNA molecules and red blood cells using optical tweezers and Raman spectroscopy [8427-86]

S. Raj, S. Rao, M. Marro Sanchez, M. Wojdyla, ICFO - Institut de Ciències Fotòniques (Spain); D. Petrov, ICFO - Institut de Ciències Fotòniques (Spain) and ICREA - Institució Catalana de Recerca i Estudis Avançats (Spain)

8427 1A Application of cheap lasers in shiffed excitation Raman difference spectroscopy [8427-45] S. Wolf, H. Döring, Hochschule Mittweida (Germany) 
8427 ID High-speed polarization-sensitive OCT at $1060 \mathrm{~nm}$ using a Fourier domain mode-locked swept source [8427-48]

S. Marschall, Technical Univ. of Denmark (Denmark); T. Torzicky, Medizinische Univ. Wien (Austria); T. Klein, W. Wieser, Ludwig-Maximilians-Univ. München (Germany); M. Pircher, E. Götzinger, S. Zotter, M. Bonesi, Medizinische Univ. Wien (Austria); B. Biedermann, LudwigMaximilians-Univ. München (Germany); C. Pedersen, Technical Univ. of Denmark (Denmark); R. Huber, Ludwig-Maximilians-Univ. München (Germany); C. Hitzenberger, Medizinische Univ. Wien (Austria); P. Andersen, Technical Univ. of Denmark (Denmark)

$8427 \mathrm{IE} \quad$ Guiding glaucoma laser surgery using Fourier-domain optical coherence tomography at $1.3 \mu \mathrm{m}[8427-49]$

M. D. Bayleyegn, H. Makhlouf, Lab. Charles Fabry, CNRS, Univ. Paris-Sud (France); C. Crotti, K. Plamann, Lab. d'Optique Appliquée, CNRS, ENSTA ParisTech, École Polytechnique (France); A. Dubois, Lab. Charles Fabry, CNRS, Univ. Paris-Sud (France)

OCT II

$8427 \mathrm{1H}$ Comparative analysis of optical coherence tomography signal and microhardness for demineralization evaluation of human tooth enamel [8427-52]

A. C. B. de Cara, D. M. Zezell, Instituto de Pesquisas Energéticas e Nucleares (Brazil); P. A. Ana, Univ. Federal do ABC (Brazil); A. M. Deana, Instituto de Pesquisas Energéticas e Nucleares (Brazil) and Univ. Nove de Julho (Brazil); M. M. Amaral, N. Dias Vieira, Jr., A. Z. de Freitas, Instituto de Pesquisas Energéticas e Nucleares (Brazil)

$8427 \mathrm{lK} \quad$ Fabrication of high quality optical coherence tomography (OCT) calibration artefacts using femtosecond inscription [8427-55]

G. C. B. Lee, J. Rasakanthan, Aston Univ. (United Kingdom); P. D. Woolliams, National Physical Lab. (United Kingdom); K. Sugden, Aston Univ. (United Kingdom)

8427 1M Coherence effects of thick objects imaging in interference microscopy [8427-57] A. A. Grebenyuk, N.G. Chernyshevsky Saratov State Univ. (Russian Federation); V. P. Ryabukho, N.G. Chernyshevsky Saratov State Univ. (Russian Federation) and Institute of Precision Mechanics and Control (Russian Federation)

\section{ADVANCED MICROSCOPIC AND SPECTROSCOPIC METHODS IV}

8427 IN Simultaneous EEG and diffuse optical imaging of seizure-related hemodynamic activity in the newborn infant brain (Invited Paper) [8427-58]

J. C. Hebden, Univ. College London (United Kingdom); R. J. Cooper, Massachusetts General Hospital (United States); A. Gibson, N. Everdell, Univ. College London (United Kingdom); T. Austin, Cambridge Univ. Hospitals NHS Trust (United Kingdom)

842710 Optimization of wavelengths sets for multispectral reflectance imaging of rat olfactory bulb activation in vivo [8427-59]

R. Renaud, M. Bendahmane, R. Chery, C. Martin, H. Gurden, F. Pain, IMNC-UMR 8165, CNRS, Univ. Paris-Sud 11 (France) 
8427 IP Fluorescence-enhanced optical spectroscopy using early arriving photons in transmission mode: a finite element approach [8427-60]

V. Piron, J.-P. L'Huillier, Lab. Arts et Métiers ParisTech Angers, Ecole Nationale Supérieure d'Arts et Métiers (France)

8427 IR Spectro-angular mapping of localized gold inclusions in Intralipid phantoms [8427-62] S. Grabtchak, Univ. of Prince Edward Island (Canada) and Dalhousie Univ. (Canada); T. J. Palmer, Univ. of Prince Edward Island (Canada); W. M. Whelan, Univ. of Prince Edward Island (Canada) and Atlantic Veterinary College (Canada)

8427 is Photoacoustic spectroscopy of weakly absorbing media using nanosecond laser pulses [8427-63]

L. A. Reis, Univ. de Coimbra (Portugal); F. A. Schaberle, Luzitin SA (Portugal); E. F. F. Silva, Univ. de Coimbra (Portugal); L. G. Arnaut, Univ. de Coimbra (Portugal) and Luzitin SA (Portugal)

\section{ADVANCED MICROSCOPIC AND SPECTROSCOPIC METHODS V}

8427 1T Infrared spectroscopic studies to understand the effect of drugs at molecular level (Invited Paper) [8427-64]

B. Singh, R. Gautam, B. Chandrasekar, S. Rakshit, V. Kumar B. N., S. Boopathy, D. Nandi,

K. Somasundaram, S. Umapathy, Indian Institute of Science (India)

$84271 \mathrm{U}$ Label-free multimodal microspectroscopic differentiation of glioblastoma tumor model cell lines combined with multivariate data analysis [8427-65]

E. Ostertag, B. Boldrini, S. Luckow, R. W. Kessler, Reutlingen Research Institute (Germany)

$84271 \mathrm{~V}$ Sensor design for cancer tissue diagnostics [8427-66]

L. La Spada, F. Bilotti, L. Vegni, Roma Tre Univ. (Italy)

$84271 \mathrm{X}$ Lithographic microfabrication of biocompatible polymers for tissue engineering and lab-on-a-chip applications [8427-68]

E. Balciunas, L. Jonusauskas, V. Valuckas, D. Baltriukiene, V. Bukelskiene, R. Gadonas, M. Malinauskas, Vilnius Univ. (Lithuania)

\section{OPTICAL THERAPY II}

842722 Toluidine blue O-conjugated gold nanoparticles for photodynamic therapy of cultured colon cancer [8427-73]

R. Al-Majmaie, N. Alattar, D. Zerulla, M. Al-Rubeai, Univ. College Dublin (Ireland)

842726 The development of skin immersion clearing method for increasing of laser exposure efficiency on subcutaneous objects [8427-77]

A. M. Kozina, E. A. Genina, G. S. Terentyuk, A. G. Terentyuk, A. N. Bashkatov, N.G. Chernyshevsky Saratov State Univ. (Russian Federation); V. V. Tuchin, N.G. Chernyshevsky Saratov State Univ. (Russian Federation), Institute of Precise Mechanics and Control (Russian Federation), and Univ. of Oulu (Finland); B. N. Khlebtsov, N.G. Chernyshevsky Saratov State Univ. (Russian Federation) and Institute of Biochemistry and Physiology of Plants and Microorganisms (Russian Federation) 
842728 Laser scattering by transcranial rat brain illumination [8427-78]

M. V. P. Sousa, Univ. de São Paulo (Brazil); R. Prates, I. T. Kato, C. P. Sabino, L. C. Suzuki, M. S. Ribeiro, Instituto de Pesquisas Energéticas e Nucleares (Brazil); E. M. Yoshimura, Univ. de São Paulo (Brazil)

842729 Accurate determination of the complex refractive index of solid tissue-equivalent phantom [8427-79]

J. Wang, Q. Ye, Z. Deng, W. Zhou, C. Zhang, J. Tian, Nankai Univ. (China)

8427 2B Comparison between experimental and computational methods for scattering anisotropy coefficient determination in dental-resin composites [8427-81]

A. Fernández-Oliveras, I. M. Carrasco, R. Ghinea, M. M. Pérez, M. Rubiño, Univ. de Granada (Spain)

8427 2C Measurements of scattering anisotropy in dental tissue and zirconia ceramic [8427-82] A. Fernández-Oliveras, O. E. Pecho, M. Rubiño, M. M. Pérez, Univ. de Granada (Spain)

8427 2D Time of correlation of low-frequency fluctuations in the regional laser Doppler flow signal from human skin [8427-85]

M. S. Folgosi-Correa, G. E. C. Nogueira, Instituto de Pesquisas Energéticas e Nucleares (Brazil)

$84272 \mathrm{E} \quad \mathrm{X}$-ray radiation-induced effects in human mammary epithelial cells investigated by Raman microspectroscopy [8427-87]

R. Risi, L. Manti, Univ. Federico II (Italy); G. Perna, M. Lasalvia, V. Capozzi, Univ. degli Studi di Foggia (Italy); I. Delfino, Univ. degli Studi della Tuscia (Italy); M. Lepore, Seconda Univ. degli Studi di Napoli (Italy)

$84272 \mathrm{G}$ Investigation of cell morphology by the TRUImagE digital holographic microscopy system [8427-89]

O. C. Chee, W. QU, K. K. Chai, Ngee Ann Polytechnic (Singapore); A. Asundi, Nanyang Technological Univ. (Singapore)

8427 2H 3D CARS image reconstruction and pattern recognition on SHG images [8427-90] A. Medyukhina, Institut für Photonische Technologien e.V. (Germany); N. Vogler, Institut für Photonische Technologien e.V. (Germany) and Friedrich-Schiller-Univ. Jena (Germany); I. Latka, Institut für Photonische Technologien e.V. (Germany); B. Dietzek, Institut für Photonische Technologien e.V. (Germany) and Friedrich-Schiller-Univ. Jena (Germany); R. Cicchi, F. S. Pavone, European Lab. for Non-linear Spectroscopy (Italy); J. Popp, Institut für Photonische Technologien e.V. (Germany) and Friedrich-Schiller-Univ. Jena (Germany)

8427 2J Diode laser based photoacoustic gas measuring instruments intended for medical research [8427-92]

A. Szabó, Á. Mohácsi, P. Novák, D. Aladzic, K. Turzó, Z. Rakonczay, G. Erős, M. Boros,

K. Nagy, G. Szabó, Univ. of Szeged (Hungary)

8427 2K Dual wavelength multiple-angle light scattering system for cryptosporidium detection [8427-93]

S. Buaprathoom, S. Pedley, S. J. Sweeney, Univ. of Surrey (United Kingdom) 
$84272 \mathrm{M} \quad$ Laser based signal and image fractal analysis for assessment of blood flow [8427-95] S. Narayanan Unni, C. Lal, Indian Institute of Technology Madras (India)

842720 Spectroscopic detection of chemotherapeutics and antioxidants [8427-97] I. Latka, R. Grüner, C. Matthäus, Institut für Photonische Technologien e.V. (Germany); B. Dietzek, Institut für Photonische Technologien e.V. (Germany) and Friedrich-Schiller-Univ. Jena (Germany); W. Werncke, Max-Born-Institut für Nichtlineare Optik und Kurzzeitspektroskopie (Germany); J. Lademann, Charité Universitätsmedizin Berlin (Germany); J. Popp, Institut für Photonische Technologien e.V. (Germany) and FriedrichSchiller-Univ. Jena (Germany)

$84272 \mathrm{P} \quad$ Laser heating of gold nanoparticles: photothermal cancer cell therapy [8427-98] N. N. Nedyalkov, P. A. Atanasov, Institute of Electronics (Bulgaria); R. A. Toshkova, E. G. Gardeva, L. S. Yossifova, M. T. Alexandrov, Institute of Experimental Morphology, Pathology and Anthropology (Bulgaria); D. Karashanova, Institute of optical materials and technologies (Bulgaria)

$84272 \mathrm{Q}$ Core-shell nanoparticles as enhanced probes for imaging applications [8427-99] M. Bloemen, W. Brullot, C. Denis, L. Vanysacker, T. Verbiest, Katholieke Univ. Leuven (Belgium)

$84272 R \quad$ Multispot two-photon imaging of mice heart tissue detecting calcium waves [8427-100] C. de Mauro, C. A. Cecchetti, D. Alfieri, Light4Tech Firenze S.r.l. (Italy); G. Borile, M. Mongillo, Venetian Institute of Molecular Medicine (Italy); F. S. Pavone, Univ. degli Studi di Firenze (Italy)

$84272 \mathrm{~V} \quad$ Improvement of axial resolution of spectral domain optical coherence tomography with wide band PLC splitter [8427-108]

J. B. Eom, Korea Photonics Technology Institute (Korea, Republic of); E. J. Min, B. H. Lee, Gwangju Institute of Science and Technology (Korea, Republic of)

$84272 Z$ Theoretical analysis of two nodal-wedges method to enhance the vibration behavior of NSOM probe with its tip being immersed partially in liquid [8427-112]

W. Lee, D.-C. Kim, B.-H. O, S.-G. Park, E.-H. Lee, S. G. Lee, Inha Univ. (Korea, Republic of)

842731 Optical biosensors utilizing polymer-based athermal microring resonators [8427-1 14] L. Wang, Dalian Univ. of Technology (China) and Univ. Gent (Belgium); X. Han, Y. Gu, H. LV,

J. Cheng, J. Teng, J. Ren, J. Wang, X. Jian, M. Zhao, Dalian Univ. of Technology (China)

842732 Analysis of skin basalioma and melanoma by multispectral imaging [8427-1 15] I. Diebele, A. Bekina, Univ. of Latvia (Latvia); A. Derjabo, J. Kapostinsh, SIA Riga Eastern Clinical Univ. Hospital (Latvia); I. Kuzmina, J. Spigulis, Univ. of Latvia (Latvia)

842734 Clinical measurements with multi-spectral photoplethysmography sensors [8427-117] L. Asare, M. Ozols, U. Rubins, O. Rubenis, J. Spigulis, Univ. of Latvia (Latvia)

$842736 \quad$ Non-linear optical imaging and fibre-based spectroscopy of fresh colon biopsies [8427-1 19] R. Cicchi, A. Sturiale, G. Nesi, D. Kapsokalyvas, F. Tonelli, F. S. Pavone, Univ. degli Studi di Firenze (Italy) 
842737 RGB imaging system for monitoring of skin vascular malformation's laser therapy [8427-120] D. Jakovels, I. Kuzmina, Univ. of Latvia (Latvia); A. Berzina, The Clinic of Laser Plastics (Latvia); J. Spigulis, Univ. of Latvia (Latvia)

842738 Full-range spectral domain optical coherence tomography using fiber-based sample scanner as self-phase shifter [8427-121]

E. J. Min, J. G. Shin, J. H. Lee, B. H. Lee, Gwangju Institute of Science and Technology (Korea, Republic of)

842739 Waveguide-type localized plasmon resonance biosensor for noninvasive glucose concentration detection [8427-122]

A. V. Nashchekin, V. N. Nevedomskiy, P. A. Obraztsov, loffe Physico-Technical Institute (Russian Federation); O. V. Stepanenko, Institute of Cytology (Russian Federation); A. I. Sidorov, State Univ. of Information Technologies, Mechanics and Optics (Russian Federation); O. A. Usov, loffe Physico-Technical Institute (Russian Federation); K. K. Turoverov, Institute of Cytology (Russian Federation); S. G. Konnikov, Ioffe PhysicoTechnical Institute (Russian Federation)

8427 3B Supercontinuum laser based double-integrating-sphere system for measuring optical properties of highly dense turbid media in the $1300-2350 \mathrm{~nm}$ region with high sensitivity [8427-124]

L. Wang, S. Sharma, B. Aernouts, H. Ramon, W. Saeys, Katholieke Univ. Leuven (Belgium)

8427 3D A multimodal holographic system for optical manipulation and injection of developing embryos [8427-126]

M. L. Torres-Mapa, M. Antkowiak, H. Cizmarova, D. E.K. Ferrier, K. Dholakia, F. Gunn-Moore, Univ. of St. Andrews (United Kingdom)

8427 3E Propagation, structural similarity, and image quality [8427-127]

J. Pérez, D. Mas, J. Espinosa, C. Vázquez, C. Illueca, Univ. de Alicante (Spain)

$84273 \mathrm{H} \quad$ Miniature wireless photoplethysmography devices: integration in garments and test measurements [8427-130]

E. Kviesis-Kipge, V. Mečņika, O. Rubenis, Univ. of Latvia (Latvia)

$842731 \quad$ Influence of low power CW laser irradiation on skin hemoglobin changes [8427-131]

I. Ferulova, J. Lesins, A. Lihachev, D. Jakovels, J. Spigulis, Univ. of Latvia (Latvia)

$84273 \mathrm{M}$ Single-layer graphene based SPR biochips for tuberculosis bacillus detection [8427-135]

N.-F. Chiu, T.-Y. Huang, C.-C. Kuo, W.-C. Lee, National Taiwan Normal Univ. (Taiwan);

M.-H. Hsieh, H.-C. Lai, Chang Gung Univ. (Taiwan)

8427 3N Development of a non-invasive LED based device for adipose tissue thickness measurements in vivo [8427-136]

K. Volceka, D. Jakovels, Z. Arina, J. Zaharans, E. Kviesis, A. Strode, E. Svampe,

L. Ozolina-Moll, M. M. Butnere, Univ. of Latvia (Latvia) 
$84273 \mathrm{P}$ FTIR characterization of animal lung cells: normal and precancerous modified e10 cell line [8427-138]

D. M. Zezell, T. M. Pereira, Instituto de Pesquisas Energéticas e Nucleares (Brazil);

G. Mennecier, L. Bachmann, Univ. de São Paulo (Brazil); A. B. Govone, Instituto de Pesquisas Energéticas e Nucleares (Brazil); M. L. Z. Dagli, Univ. de São Paulo (Brazil)

$84273 Q \quad$ Corneal topography reinterpretation through separate analysis of the projected rings [8427-139]

J. Espinosa, A. B. Roig, D. Mas, C. Hernández, C. Illueca, Univ. de Alicante (Spain)

$84273 R \quad$ In-vivo real-time monitoring of nanoparticle clearance rate from blood circulation using high speed flow cytometry [8427-140]

M. Sarimollaoglu, D. A. Nedosekin, E. I. Galanzha, V. P. Zharov, Univ. of Arkansas for Medical Sciences (United States)

$84273 \mathrm{U}$ Optimal irradiation condition of demineralized dentin treatment with a nanosecond pulsed laser at $5.8 \mu \mathrm{m}$ wavelength range [8427-143]

K. Ishii, M. Saiki, T. Kita, Osaka Univ. (Japan); K. Yoshikawa, K. Yasuo, K. Yamamoto, Osaka Dental Univ. (Japan); K. Awazu, Osaka Univ. (Japan)

$84273 \mathrm{X} \mathrm{Al(III),} \mathrm{Pd}(\mathrm{II})$, and $\mathrm{Zn}(\mathrm{II})$ phthalocyanines for inactivation of dental pathogen Aggregatibacter actinomycetemcomitans as planktonic and biofilm-cultures [8427-146] V. Kussovski, The Stephan Angeloff Institute of Microbiology (Bulgaria); V. Mantareva, I. Angelov, Institute of Organic Chemistry with the Ctr. of Phytochemistry (Bulgaria); L. Avramov, Institute of Electronics (Bulgaria); E. Popova, Medical Univ. Plovdiv (Bulgaria); S. Dimitrov, Medical Univ. Sofia (Bulgaria)

8427 3Y Stereomicroscopic evaluation of the joint cartilage and bone tissue in osteoporosis [8427-147]

L. Vasile, Univ. de Medicina si Farmacie Victor Babes, Timisoara (Romania); R. Torok, Univ. de Vest din Timisoara (Romania); B. Deleanu, County Hospital Timisoara (Romania);

C. Marchese, A. Valeanu, R. Bodea, Univ. de Medicina si Farmacie Victor Babes, Timisoara (Romania)

$84273 Z$ Femtosecond irradiation of chicken corneas analyzed by digital holographic microscopy [8427-148]

A. Fimia, M. Gomariz, A. Murciano, P. Acebal, R. Madrigal, L. Carretero, Univ. Miguel Hernández de Elche (Spain); J. L. Alió, A. Rodriguez, VISSUM Corp. (Spain); E. Fernández, Univ. Miguel Hernández de Elche (Spain)

842740 Stereomicroscopic study of the human tooth caries: clinical and morphological correlations [8427-149]

R. Oancea, L. Vasile, C. Marchese, R. Sava-Rosianu, Univ. de Medicina si Farmacie Victor Babes, Timisoara (Romania)

842741 Electronomicroscopic evaluation of the microlesional aspects in the pulp dentinal complex after repeated whitening therapy [8427-150]

R. Bodea, R. Jianu, C. Marchese, L. Vasile, Univ. de Medicina si Farmacie Victor Babes, Timisoara (Romania) 
842743 Effects of the position of galactose units to $\mathrm{Zn}$ (II) phthalocyanine on the uptake and photodynamic activity towards breast cancer cells [8427-152]

V. Mantareva, Institute of Organic Chemistry with the Ctr. of Phytochemistry (Bulgaria);

A. Kril, Institute of Experimental Morphology, Pathology and Anthropology with Museum (Bulgaria); I. Angelov, Institute of Organic Chemistry with the Ctr. of Phytochemistry (Bulgaria); R. Dimitrov, Institute of Biology and Immunology of Reproduction (Bulgaria); E. Borisova, L. Avramov, Institute of Electronics (Bulgaria)

842744 Photodynamic therapy with water-soluble phtalocyanines against bacterial biofilms in teeth root canals [8427-153]

R. Gergova, T. Georgieva, Medical Univ. Sofia (Bulgaria); I. Angelov, V. Mantareva, Institute of Organic Chemistry with the Ctr. of Phytochemistry (Bulgaria); S. Valkanov, Institute of Metal Science (Bulgaria); I. Mitov, S. Dimitrov, Medical Univ. Sofia (Bulgaria)

842746 Photonic crystal fibers for food quality analysis [8427-155]

A. V. Malinin, N.G. Chernyshevsky Saratov State Univ. (Russian Federation) and SPE Nanostructed Glass Technology (Russian Federation); A. A. Zanishevskaja, N.G. Chernyshevsky Saratov State Univ. (Russian Federation); V. V. Tuchin, N.G. Chernyshevsky Saratov State Univ. (Russian Federation), Institute of Precise Mechanics and Control (Russian Federation), and Univ. of Oulu (Finland); Yu. S. Skibina, N.G. Chernyshevsky Saratov State Univ. (Russian Federation) and SPE Nanostructed Glass Technology (Russian Federation); I. Yu. Silokhin, SPE Nanostructed Glass Technology (Russian Federation)

842747 Monitoring of the microhemodynamic in an aggressive clinical behavior of cerebral hemorrhage using dynamic light scattering techniques [8427-156]

M. A. Vilensky, O. V. Semyachkina-Glushkovskaya, P. A. Timoshina, V. A. Berdnikova, Y. V. Kuznetsova, I. A. Semyachkin-Glushkovsky, D. N. Agafonov, N.G. Chernyshevsky Saratov State Univ. (Russian Federation); V. V. Tuchin, N.G. Chernyshevsky Saratov State Univ. (Russian Federation), Institute of Precise Mechanics (Russian Federation), and Univ. of Oulu (Finland)

842748 Porosity at photo-induced fat cell lipolysis [8427-157]

V. A. Doubrovsky, Saratov State Medical Univ. (Russian Federation); I. Yu. Yanina, Saratov State Medical Univ. (Russian Federation) and N.G. Chernyshevsky Saratov State Univ. (Russian Federation); V. V. Tuchin, N.G. Chernyshevsky Saratov State Univ. (Russian Federation), Institute of Precise Mechanics (Russian Federation), and Univ. of Oulu (Finland)

$84274 \mathrm{~B} \quad$ Superconducting nanowire single-photon detectors for picosecond time resolved spectroscopic applications [8427-160]

T. May, J. Toussaint, R. Grüner, M. Schubert, H.-G. Meyer, B. Dietzek, J. Popp, Institut für Photonische Technologien e.V. (Germany); M. Hofherr, K. Il'in, D. Henrich, M. Arndt, M. Siegel, Karlsruher Institut für Technologie (Germany)

8427 4C Spectrally resolved anti-Brownian electrokinetic (ABEL) trapping of single peridininchlorophyll-proteins in solution [8427-161]

S. Bockenhaver, Q. Wang, W. E. Moerner, Stanford Univ. (United States) 
$84274 D$ Anti-Stokes effect CCD camera and SLD based optical coherence tomography for full-field imaging in the $1550 \mathrm{~nm}$ region [8427-162]

L. Kredzinski, M. J. Connelly, Univ. of Limerick (Ireland)

Author Index 


\title{
Conference Committee
}

\author{
Symposium Chairs
}

Francis Berghmans, Vrije Universiteit Brussel (Belgium)

Ronan Burgess, European Commission (Belgium)

Jürgen Popp, Institut für Photonische Technologien e.V. (Germany)

Peter Hartmann, SCHOTT AG (Germany)

Honorary Symposium Chair

Hugo Thienpont, Vrije Universiteit Brussel (Belgium)

\section{Conference Chairs}

Jürgen Popp, Institut für Photonische Technologien e.V. (Germany)

Wolfgang Drexler, Medizinische Universität Wien (Austria)

Valery V. Tuchin, N.G. Chernyshevsky Saratov State University (Russian Federation), Institute of Precision Mechanics and Control (Russian Federation), and University of Oulu (Finland)

Dennis L. Matthews, UC Davis Medical Center (United States)

\section{Programme Committee}

Peter E. Andersen, Technical University of Denmark (Denmark)

Arthur E. T. Chiou, National Yang-Ming University (Taiwan)

Paul Garside, University of Glasgow (United Kingdom)

Markus Sauer, Universität Bielefeld (Germany)

Ernst H. K. Stelzer, Goethe-Universität Frankfurt am Main (Germany)

Hugo Thienpont, Vrije Universiteit Brussel (Belgium)

Siva Umapathy, Indian Institute of Science (India)

Alex I. Vitkin, Ontario Cancer Institute (Canada)

Gert von Bally, Center for Biomedical Optics and Photonics (Germany)

Brian C. Wilson, Princess Margaret Hospital (Canada)

Session Chairs

1 Microscopy I

Jürgen Popp, Institut für Photonische Technologien e.V. (Germany)

2 Microscopy II

Rainer Heintzmann, Institut für Photonische Technologien e.V.

(Germany) and Friedrich-Schiller-Universität Jena (Germany) 
Malte C. Gather, Technische Universität Dresden (Germany)

4 Optical Sensors II

Tia E. Keyes, Dublin City University (Ireland)

5 Optical Micromanipulation I

Andreas Ostendorf, Ruhr-Universität Bochum (Germany)

6 Optical Micromanipulation II

James Chan, NSF Center for Biophotonics Science and Technology, University of California, Davis (United States)

$7 \quad$ Advanced Microscopic and Spectroscopic Methods I

Thomas G. Mayerhöfer, Institut für Photonische Technologien e.V. (Germany)

8 Advanced Microscopic and Spectroscopic Methods II Francesco S. Pavone, Universitá degli Studi di Firenze (Italy)

9 Advanced Microscopic and Spectroscopic Methods III Roberto Pini, Instituto di Fisica Applicata "Nello Carrara" (Italy)

OCT I

Peter E. Andersen, Technical University of Denmark (Denmark)

11 OCTII

Alex I. Vitkin, Ontario Cancer Institute (Canada)

12 Advanced Microscopic and Spectroscopic Methods IV

Siva Umapathy, Indian Institute of Science (India)

13 Advanced Microscopic and Spectroscopic Methods $V$

Jeremy C. Hebden, University College London (United Kingdom)

14 Optical Therapy I

Valery V. Tuchin, N.G. Chernyshevsky Saratov State University (Russian Federation), Institute of Precision Mechanics and Control (Russian Federation), and University of Oulu (Finland)

15 Optical Therapy II

Elena V. Zagaynova, Nizhny Novgorod State Medical Academy (Russian Federation) 